\title{
River Analyser - Multiresidue Immunoanalytical Monitoring Tools
}

\section{Andreas Brecht', Claudia Barzen', Albrecht Klotz', Günter} Gauglitz', Richard Harris' ${ }^{2}$, Geoffrey Quigley' ${ }^{2}$, James Wilkinson', Soizic Fraval ${ }^{3}$, Pascale Sztajnbok ${ }^{3}$, Damia Barceló ${ }^{4}$, Jordi Gascón', Michael Steinwand ${ }^{5}$, Ram Abuknesha ${ }^{6}$

'Institute of Physical Chemistry. University of Tübingen. 72076 Tübingen, FRG ${ }^{2}$ Optoelectronics Research Centre. University of Southampton, Southampton SO17 1BJ. Great Britain

${ }^{3}$ Anjou Recherche / Compagnie Générale des Eaux, Chemin de la Digue BP 76, 78603 Maisons-Laffitte, France

${ }^{4}$ Centro De Investigacion Y Desarrollo, Jordi Girona 18 - 26. 08034 Barcelona, Spain ${ }^{5}$ Perkin-Elmer GmbH. Bodenseewerk. 88647 C̈berlingen. FRG ${ }^{6}$ GEC Marconi Materials Technology. Elstree Way, Borehamwood, Herfordshire WD6 IRX, Great Britain

Abstract. Immunoanalytical techniques find growing acceptance in the field of environmental monitoring (Van Emon and Gerlach 1995). Although immunoassays have many attractive features, the lack of multiresidue approaches is a serious obstacle in environmental monitoring. In many environmental situations the simultaneous determination of more than one analyte is required. The situation is further complicated by the fact, that the analyte panels. which are to be detected, may vary with location and season.

The RIANA project (RIver ANAlyser) is focused on immunoanalytical tools, that allow to detect multiple analytes in a single run. The application areas foreseen 
are online monitoring - e.g. at pumping sites or transportable devices for field use. In this paper we describe the concept and give first results.

\section{Introduction}

Rivers, throughout Europe. form a major source of process water as well as raw water for human consumption. European rivers, however, collect a significant proportion of both treated and untreated waste water from sewage plants and industro-agricultural processes. This. as well as the use of rivers as waterways for transportation, yields to a ver. complex environment which is prone to pollution. This environment is subsequently transferred to the estuarine and coastal sea areas. The situation is aggravated in basins with reduced water exchange such as the Mediterranean and the Baltic Seas where pollution by rivers can cause serious environmental problems affecting human health and the economical prospects of coastal activities.

The role of rivers as a vital source of drinking water as well as for industrial/ agricultural processes necessitates continuous monitoring and control of the water quality (Meulenberg and Stoks 1995). This monitoring should be effected at pumping sites. industrial outputs and various important interfaces. In essence. the health of major rivers should be monitored continuously and regularly from source to coast. This implies that concentrations and transition/ life-cycles of pollutants in rivers should be monitored and effective control established.

The objective of the RIANA project is to develop a system for monitoring organic compounds present in polluted river water and in surface water. The system will be a multiresidue bioanalytical device. allowing to test in one sample a multitude of simultaneously present agents. The system envisaged is expected to be more cost effective than established reference techniques. to allow the detection of a broader range of analytes with a single device than with established reference techniques. and to avoid most of the sample pre-treatment common in current environmental 
multiresidue analysis with concomitant advantages in speed. The multianalyte approach pursued will overcome one of the major limitations of immunoanalytical techniques in environment - the limitation of a single analyte per test. An overview of the main building blocks is given in Figure 37.

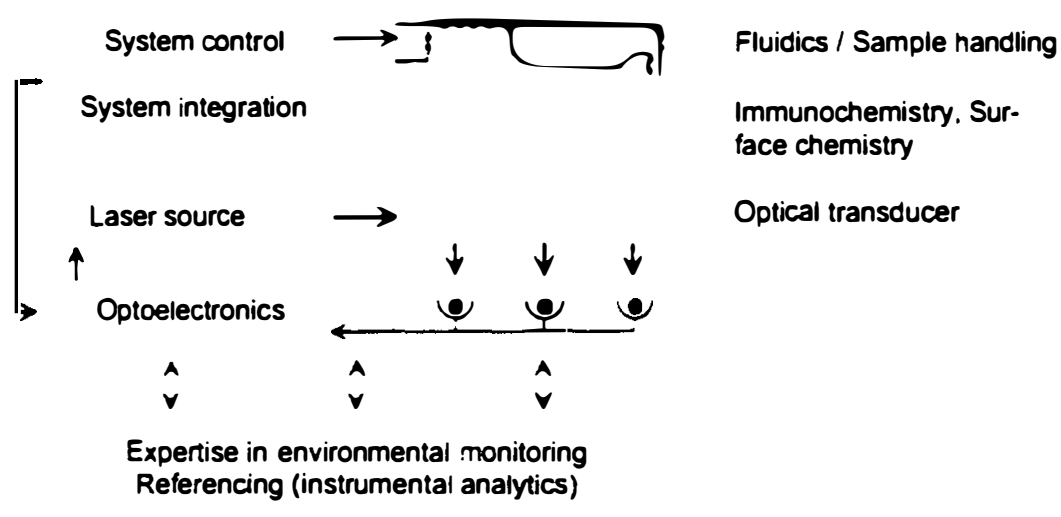

Figure 37: Building blocks of the RIAN.A approach. Vajor building blocks and research topics are immunochemistry, surface chemistry, and the optical transducer. Fluidics. optoelectronics and system control serve to "glue" the system together. Referencing to established techniques is a central part of the project

\section{Materials, methods and concepts}

\section{Transducer}

The transducer system is based on fluorescence detection (Wadkins et al. 1995). Targets are effective excitation and spatially resolved detection of fluorescence labelled antibodies bound to the transducer surface. Initially a simple bulk-optical device is used. This will be replaced by an integrated optical transducer element which will lead to reduced active area and improved excitation and detection. For each analyte to be detected, a defined spot of the transducer surface is modified with a 


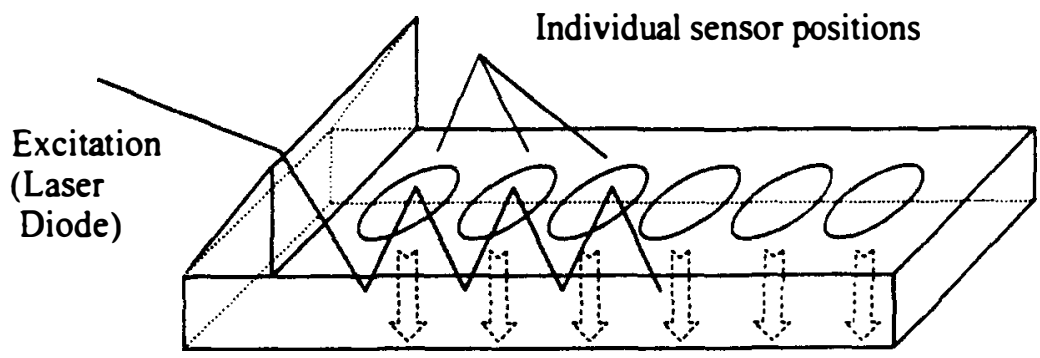

To detection system

(fiber coupling)

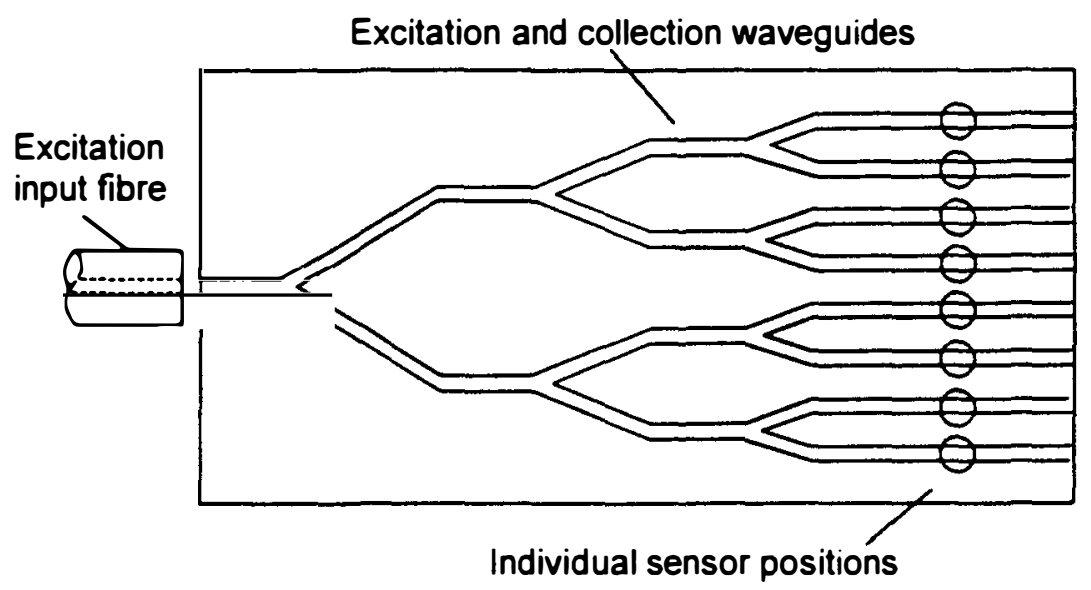

Figure 38: Optical multispot transducers investigated within the RIANA project. Top: simple bulk optical transducer - detection spots are about $1.5 \mathrm{~mm} * 1.5 \mathrm{~mm}$ in size; Bontom: integrated optical channel waveguide device - detection spots are about $0.1 \mathrm{~mm} * 5 \mathrm{~mm}$ in size

derivate of the respective analyte (Figure 38). Transducer design starts with numerical modelling of different structures. starting from monomode waveguides. modified by high index cover layers to optimise fluorescence excitation. The waveguide model developed to analyse the first generation integrated optical ( $\mathrm{IO}$ ) sensor uses a transfer matrix approach to allow the analysis of multilayer structures. The argument principle method is used to determine the number and effective indices of modes which exist in 
the multilayer region of the sensor. A more detailed description of the model is given by Harris and Wilkinson (1995).

\section{Immunochemistry}

Antibodies will be used to bind selectively to the analytes of choice. Antibodies will further be used as components of a flexible immobilisation system. that allows the selection of the transducer surface on-line during the assay (Brecht and Abuknesha 1995. Abuknesha and Brecht 1997). The performance of immunochemistry is assessed in a conventional ELISA testbed (Tijssen 1985).

Table 18: Target analytes selected for the RIANA project

\begin{tabular}{ll}
\hline Toluene & Pentachlorophenol \\
2.4.5-Trichlorophenol & 2.4.6-Trichlorophenol \\
Hydroxycarbofuran & Desethylatrazine \\
Atrazine & Simazine \\
2.4-D & Methy.lchlorophenoxypropionic \\
& acid \\
Alachlor & Glyphosate \\
Isoproturon & Non!lphenol \\
\hline
\end{tabular}

For the detection of analıtes a competitive test scheme will be implemented. ELISA results will serve as a reference. Competition will occur between the free analyte and compounds of the biochemical immobilisation system conjugated with analyte derivatives. The detection will occur in a heterogeneous format. i. e. at the transducer surface. The analytes addressed within the project cover pesticides as well as industrial pollutants (Table 18). By purpose a set of compounds was selected, which can not be determined within a single chromatographic run by conventional 


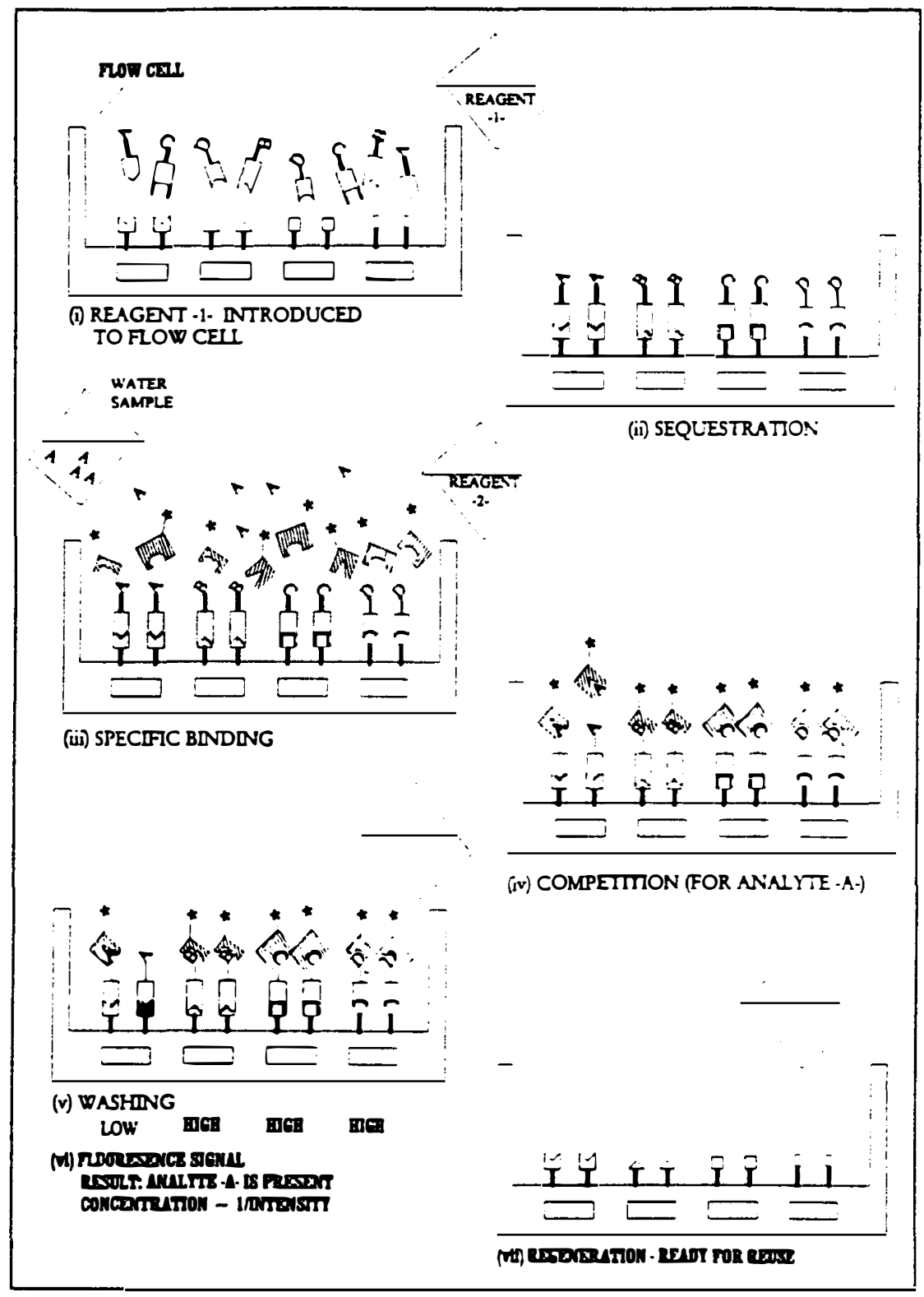

Figure 39: Assay concept. Antibody conjugates serve as "auxiliary immobilising system" and bring analyte specific chemistry to selected areas at the transducer surface. Sequential and nonsequential assay concepts are possible 
instrumental analysis. Also polar and acidic compounds were included, which may escape typical extraction procedures (Chiron and Barceló 1993).

Immunogens were made by coupling selected analyte and auxiliary ligand derivatives to mixtures of bovine serum albumin. key hole limpet haemocyanine and fetuin. Antibody activity in the various antisera was assessed by antibody dilution response curve experiments in which immobilised hapten-protein conjugates were used to assess antibody titre in an ELISA format.

An auxiliary immobilising system consisting of antibodies specific for haptens without environmental relevance will serve to target the detection compounds to selected areas of the transducer surface (Figure 39). The spatially resolved immobilisation of the haptens of the auxilian system is achieved by inkjet n.pe microdrop delivery systems (Blanchard et al. 1996).

Table 19: Reference sample matrix used in the RIANA project

\begin{tabular}{lc}
\hline Parameter & Value \\
\hline $\mathrm{pH}$ & 7.5 \\
\hline Conductivity $(\mathrm{mS} / \mathrm{m})$ & 50 \\
\hline Total Hardness $(\mathrm{mg} \mathrm{CaCO} 3 ! \mathrm{l})$ & 200 \\
\hline Chloride $(\mathrm{mg} / \mathrm{l} \mathrm{Cl})$ & 50 \\
\hline Sulphates $(\mathrm{mg} / \mathrm{SO} 4)$ & 40 \\
\hline Silica $(\mathrm{mg} / \mathrm{l} \mathrm{SiO} /)$ & 10 \\
\hline Magnesium $(\mathrm{mg} / \mathrm{l})$ & 20 \\
\hline Potassium $(\mathrm{mg} /)$ & 10 \\
\hline Sodium $(\mathrm{mg} / \mathrm{l})$ & 50 \\
\hline Nitrates $(\mathrm{mg} / \mathrm{l})$ & 20 \\
\hline Humic acids $(\mathrm{mg} / \mathrm{l})$ & 4 \\
\hline
\end{tabular}

\section{Reference analysis and validation}

Validation is carried out by laboratories with a high degree of experience in analysing complex environmental samples. Where standardised methods do not exist neither at 
the national level nor at the European level, US-EPA protocols will be adapted (Marco et al. 1995). A first step in referencing the RIANA tools is comparative studies with ELISA type assays developed with the RIANA immunochemistry. Later on the RIANA analyser hardware will be compared to instrumental analytical techniques. Real world samples from sampling campaigns will be used. Initial trials and calibrations are carried out in a sample matrix matched to values typical for European water samples (Table 19).

\section{Results and discussion}

\section{Transducer}

The efficiency of fluorescence excitation of bulk optical and integrated optical structures can be significantly improved by the introduction of thin high index cover
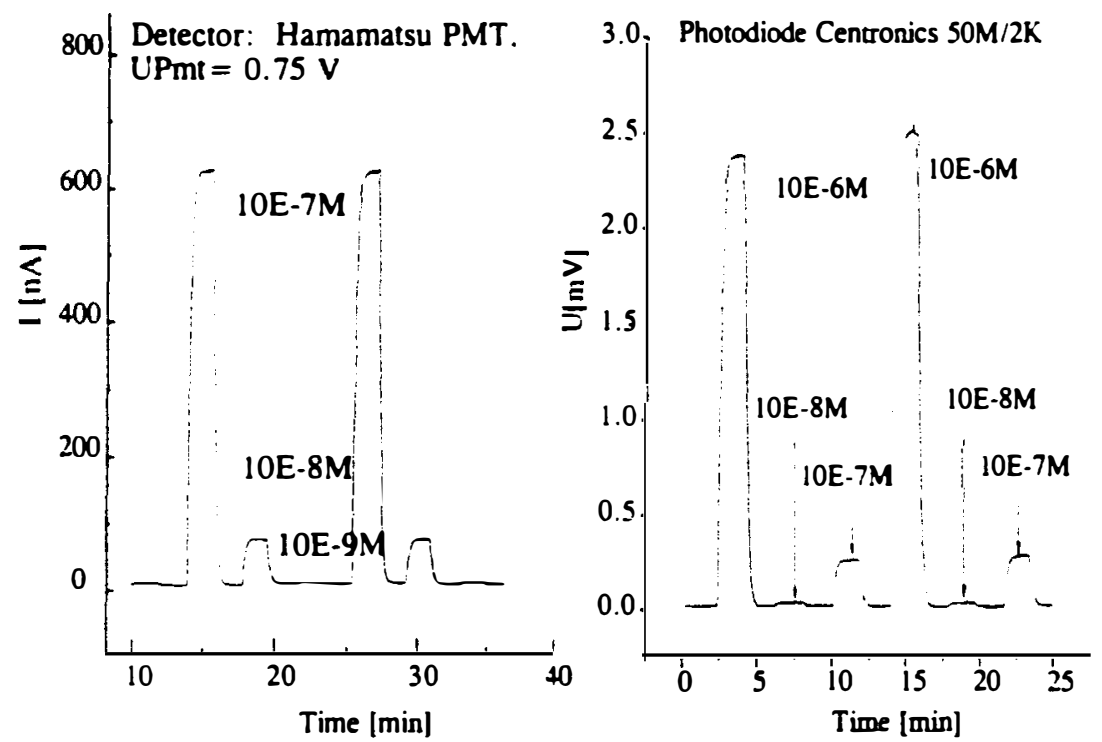

Figure 40: Detection of fluorophore solutions with bulk optical transducer. Concentrations of $10^{-4} \mathrm{M}$ can be detected with both. photomultiplier and solid state detectors. This corresponds to $0.01 \%$ of a monolayer of fluorescence labelled antibodies. The photomultiplier is one order of magnitude more sensitive than the photodiode 


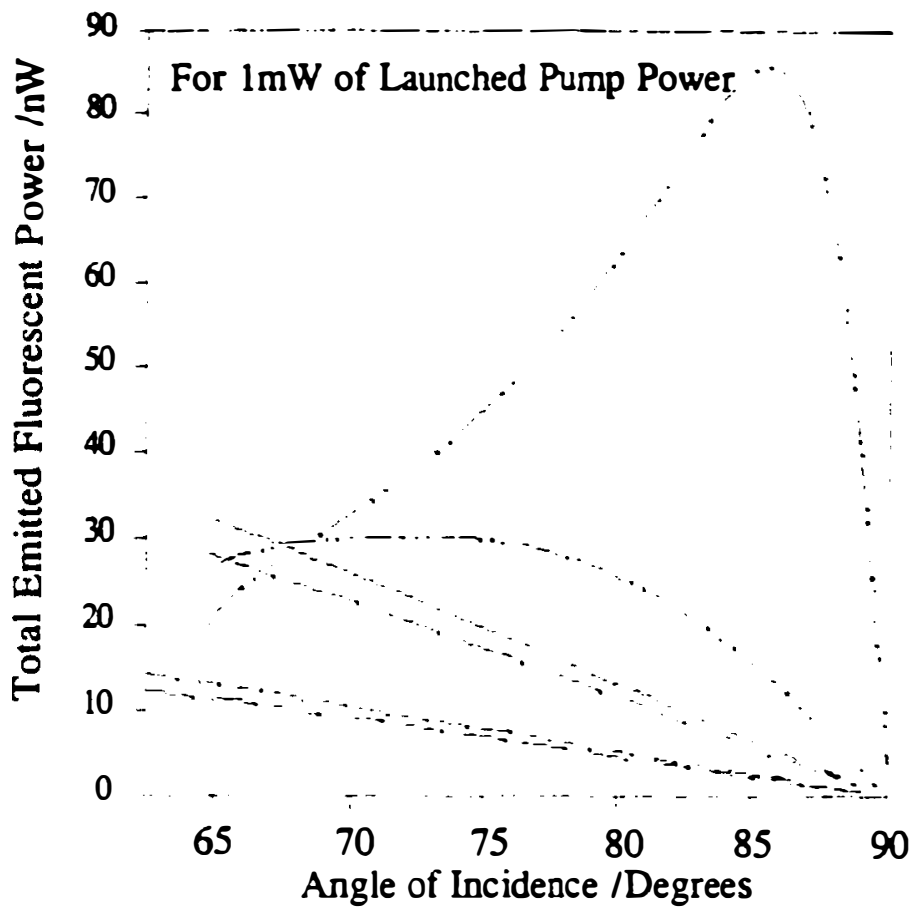

$$
\begin{aligned}
& \text { Pransdice }=1.47: \text { TE Polarisation } \\
& \text { Pransdice }=1.47: \text { TM Polarisation } \\
& \text { - Rtanstice }=1.76: \text { TE Pobrisation } \\
& \text { Rtranstice }=1.76: \text { TM Polar sation } \\
& \text { Rransdice }=1.47 \text { + High I adex Layer : TE Polarisation }
\end{aligned}
$$

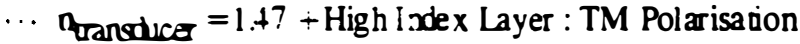

Figure 41: Total emitted fluorescent power from a mor.olayer of dye-modified antibodies adsorbed to the surface of various bulk optical sensor strictures

layers on top of the substrate or waveguide. The sensitivity of bulkoptical sensor structures. with and without high-index films, is plotted in Figure 41. Model 
calculations indicate similar behaviour for monomode channel waveguide designs. High index coated waveguide transducers were manufactured and are currently being tested. During a test cycle the formation of up to $1 \%$ of an antibody monolayer is expected at the transducer surface. This surface coverage can be converted to a corresponding bulk fluorophore concentration, indicating, that the transducer should be capable of detecting $10^{-8} \mathrm{M}$ fluorophore solutions. This could be demonstrated for a long wavelength excitable dye (Amersham Cy5.5) with the bulk optical transducer (Figure 40).

\section{Immunochemistry}

Titration tests have demonstrated detectable antibod! activity to analyte derivatives in all samples tested. The detectable antibody binding activity levels showed considerable variation with different structures. As expected. antisera to small analytes

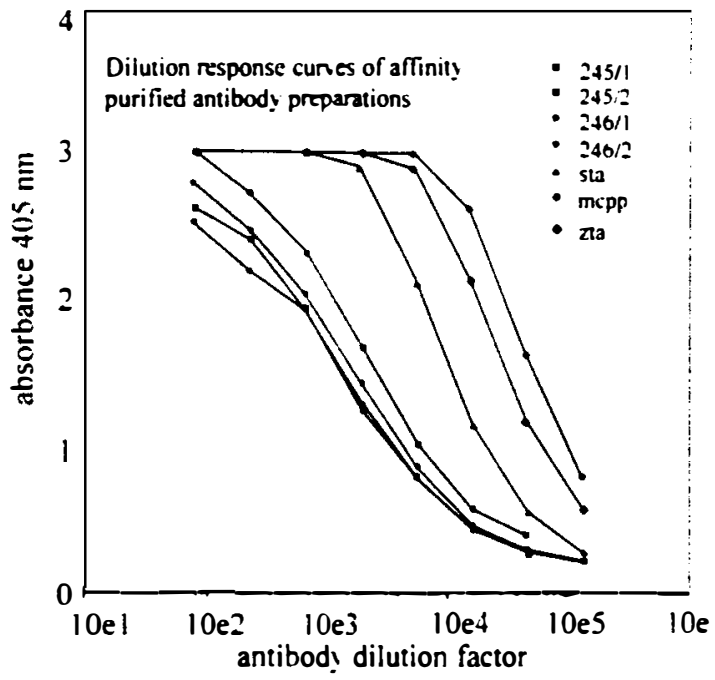

Figure 42: Antibody dilution response curves of affinity purified antibody preparations. The tests were carried out by ELISA using antibody stocks of $10 \mathrm{mg} / \mathrm{ml}$. The graphs show the substantial differences between the antibody titres of the various antibody preparations. 2.4.5 $=2.4 .5$ Trichlorophenol; $2,4,6=2,4,6$-Trichlorophenol; $\mathrm{mcpp}=$ Methylchlorophenoxypropionicacid; $\mathbf{s t a}=$ Simazine-thioaceticacid; $z$ ta $=$ atrazine-thioaceticacid 
such as toluene. glyphosate and nonylphenol showed lower levels of activities than the larger chlorophenol haptens (Figure 42). Antisera to auxiliary ligands showed much greater levels of binding activities by comparison to the responses to the panel of analytes. This is due to the relatively larger molecular weights of the selected auxiliary ligands and more antigenic structural features of these substances.
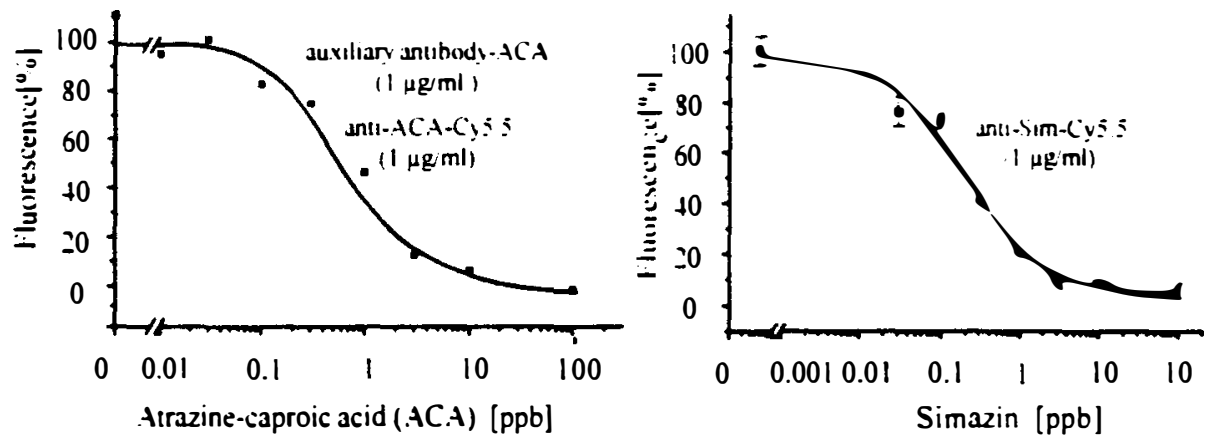

Figure 43: Proof of principle for auxiliary immobitising system. Left: calibration for a s-triazine derivative with auxilian słstem. Right: ialibration for a s-triazine derivative without auxiliañ system

The bulk optical transducer was used to implement first fluoro-immunoassays. The transducer surface was modified with an amino-dextran layer to reduce non specific binding. To this layer either an analıte derivate or an auxiliary hapten was coupled by carbodiimide chemistry (Piehler et al. 1996). Antibod! concentrations were adjusted between $0.5 \mu \mathrm{g} / \mathrm{ml}-1 \mu \mathrm{g} / \mathrm{ml}$. Aralyte specific antibodies were labelled by $C y .5$ with a ratio of about 2 fluorophores per antibody molecule. A flow injection analyser was used to deliver the sample solution to the transducer surrace. Increasing amounts of free analyte were added to suppress the antibody binding to the surface. With both systems calibration curves were obtained (Figure 43). Individual assays could be run within $10-15$ minutes. In both cases test midpoints below I ppb could be reached. It must be noted, that s-triazine conjugates represent relatively good immunogens. The detection with the bulk optical transducer at the concentrations 
selected $(0.5-1 \mu \mathrm{g} / \mathrm{ml}$ antibody) was possible at a signal to noise ratio of about 100 . This is sufficient. but further reduction of antibody concentrations would require improved detection systems.

\section{Reference analysis and validation}

The ELISA type assays are a first tool for assessment of immunochemistry performance. An important parameter is the cross reactivity pattern obtained for different antibody preparations. In Figure 44 the cross reactivity of an anti-atrazine antibody against two other s-triazines is shown. Not unexpectedly cross reactivity is considerable. The test midpoints are between 1 and $10 \mathrm{ppb}$.

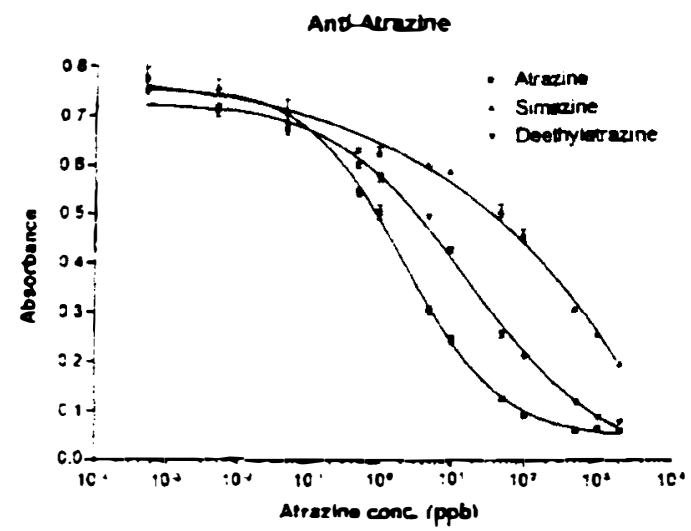

Figure 44: Assessment of crossreactivity for analyte specific antibody

\section{Outlook}

The first results of the RIANA project are encouraging. Immune responses against a uide range of environmentally relevant substances were obtained. The transfer of results from ELISA to the optical transducer works. With high affinity antibodies test midpoints below $1 \mathrm{ppb}$ can be reached. Improved optical detection systems will allow the further optimisation of assay performance. 
The use of ELISA type assays as a testbed for the assessment of immunochemistry performance is a pragmatic, and effective approach. The data will not directly scale to the response from the optosensor setup. but the general tendency of performance ian be estimated.

The immune responses obtained for various antigens indicate. that it will be difficult to obtain this high level of performance for all the analytes. The careful optimisation of the hapten used for production of the auxiliary tracer conjugate may allow to do some optimisation in these cases. Also cross reactivity may preclude the individual substance identification in some cases. Sum parameters however should be readily obtainable.

So far the auxiliary immobilising system could be shoun to work. However. this was tested with a single analyte under investigation. The scale up to 5 or 6 analytes within one sample will make the picture more complicated. For 5 analytes 10 different antibodies have to be used ( 5 analyte specific. 5 auxiliary). This will inevitably complicate the system and require thorough optimisation.

The data presented here were obtained within less than one year. We hope to come close to a workable system within the entire project duration of 3 years.

\section{Acknowledgement}

The RIANit project is funded under the Environment And Climate Programme 1994 1998 / (Area 2) En ironmental Technologies (Instruments, Techniques And Methods For Monitoring The Environment) ENV4-CT95-0066 


\section{References}

Abuknesha. R.. Brecht, A. (1997): Multi-analste immunoanalysis: Practical aspects of manufacturing transducers. Biosens. Bioelectron. 3. 159-160.

Blanchard. A.P.. Kaiser. R.J., Hood, L.E. (1996): High-density oligonucleotide arrays. Biosens. Bioelectron. 11 (6-7), 687-690.

Brecht. A., Abuknesha, R. (1995): Multi-analyte immunoassays application to environmental analysis. Trends Anal. Chem. 14(7), 361-371.

Chiron. S., Barceló, D. (1993): Determination of pesticides in drinking water by online solid-phase disk extraction followed by various liquid chromatographic systems. J. Chromatogr. 645(1), 125-133.

Harris. R.D.. Wilkinson. J.S. (1995): Waveguide surface plasmon resonance sensors. Sensors and Actuators B 29. 261-267.

Marco. M.-P.. Chiron. S.. Gascón. J.. Hammock. B.D.. Barceló. D. (1995): Validation of two immunoassay methods for environmental monitoring of carbaryl and 1naphthol in ground water samples. Anal. Chim. Acta 311. 319-329.

Meulenberg. E.P.. Stoks. P.G. (1995): Water quality control in the production of drinking water from river water. Anal. Chim. Acta 311. 407-413.

Piehler. J.. Brecht. A.. Geckeler. K.E.. Gauglitz. G. (1996): Surface modification for direct immunoprobes. Biosens. Bioelectron. 11(6-7), 579-590.

Tijssen. P. (1985): Practice and theory of enzyme immunoassays. Elsevier. Amsterdam.

Van Emon. J.M.. Gerlach. C.L. (1995): The right environment for the immunoassay. Chemtech 11. 51-54.

Wadkins. R.M.. Golden. J.P.. Ligler. F.S. (1995): Calibration of biosensor response using simultaneous evanescent wave excitation of cyanine-labelled capture antibodies and antigens. Anal. Biochem. 232 (1). 73-78. 